\title{
Comparative Analysis of Existing and Alternative Version of the Special Theory of Relativity
}

\author{
Alexander Alexandrovich Antonov \\ Independent Researcher, Kiev, Ukraine \\ Email: telan@bk.ru
}

How to cite this paper: Antonov, A.A. (2020) Comparative Analysis of Existing and Alternative Version of the Special Theory of Relativity. Journal of Modern Physics, 11, 324-342.

https://doi.org/10.4236/jmp.2020.112020

Received: January 25, 2020

Accepted: February 21, 2020

Published: February 24, 2020

Copyright (๑) 2020 by author(s) and Scientific Research Publishing Inc. This work is licensed under the Creative Commons Attribution International License (CC BY 4.0).

http://creativecommons.org/licenses/by/4.0/

\section{(c) (i) Open Access}

\begin{abstract}
The article explains that: 1) relativistic formulas obtained in the existing version of the special theory of relativity (STR) are incorrect; 2) relativistic formulas obtained in the existing version of the STR are explained incorrect due to the use of the nonexistent in nature principle of light speed non-exceedance; 3 ) conclusions on physical unreality of imaginary numbers and existence of only our visible Monoverse drawn from relativistic formulas of the existing version of the STR are incorrect due to the use of the incorrect principle of light speed non-exceedance. In other words, the existing version of the STR created in the 20th century is not quite true. Moreover, the correct STR could not be created in the 20th century, since 1) the principle of physical reality of imaginary numbers refuting experimentally the postulated (i.e. being an unproven assumption) principle of light speed non-exceedance was published only in the $21^{\text {st }}$ century; 2 ) experimental data whose mathematical analysis discerned the quaternion structure of the hidden Multiverse consisting of twenty to twenty-two invisible parallel universes in six-dimensional space were obtained by WMAP and Planck spacecraft only in the $21^{\text {st }}$ century; 3 ) explanation of the way how astronomical observations of constellations of the starry sky in portals can experimentally prove the existence of invisible universes was published only in 2019. Therefore, the article presents an alternative version of the STR, free from the shortcomings of its existing version. Other relativistic formulas that have been obtained in the alternative version of the STR are explainable both at sub-light and hyper-light speeds, and for real and imaginary values of all quantities corresponding to these formulas. Therefore, the principle of light speed non-exceedance is excluded from this version of the STR. For the same reason, the alternative version of the STR states that there is a Multiverse of mutually invisible parallel universes, rather than a Monoverse, since all the mutually invisible parallel universes are relative to
\end{abstract}


each other beyond the event horizon. It also explains how the existence of these invisible parallel universes can be proved by astronomical observations in portals. Moreover, the WMAP and Planck spacecraft data are used in the alternative version of the STR to clarify the structure of the hidden Multiverse. Their mathematical processing has testified that the hidden Multiverse has a quaternion structure and contains twenty-twenty two invisible universes in six-dimensional space.

\section{Keywords}

Imaginary Numbers, Special Theory of Relativity, Dark Matter, Dark Energy, Dark Space, Multiverse, Hyperverse

\section{Introduction}

The existing version of the special theory of relativity (STR) [1] [2] [3] created in the $20^{\text {th }}$ century is a great scientific achievement of physics. But its creators Joseph Larmor [4], Nobel Prize laureate Hendrik Antoon Lorentz [5], Jules Henri Poincaré [6], Nobel Prize laureate Albert Einstein [7] and other outstanding scientists were in advance of their time and could not complete the theory, since they began to develop a theory for completion which in physics there was no necessary knowledge at that time. Therefore STR creators had to replace this missing knowledge with postulates, i.e. unproven assumptions. In other words, they had to guess this knowledge. But they did not guess. As a result, the version of the STR that was created in the $20^{\text {th }}$ century and still exists is unfinished. Therefore, the STR was not actually created in the $20^{\text {th }}$ century ${ }^{1}$. In fact, at that time only a task of its creation was set and an attempt to find at least its partially correct solution was made.

Nevertheless, such an unfinished version of the STR is a great scientific achievement of Albert Einstein and other authors of SRT, since it induced physical community to persistent efforts in solving the problem of creating the correct STR.

\section{The Logic of Reasoning That Led to Creation of the Existing Version of the STR}

Before proceeding to consideration of shortcomings of the existing version of the STR, it would be useful to understand the logic of its creation and the circumstances in which it was created. Then it would become clear that the STR version created in the $20^{\text {th }}$ century was a great scientific achievement, and its errors couldn't be avoided at that time.

First of all, creating the STR it should be determined what space we lived in. However, we have not the slightest idea about other space, than the space of our

${ }^{1}$ We can say that Albert Einstein laid the foundation of SRT and began to build the building of this theory, but due to the lack of necessary experimental data this building was not completed. 
home and the nature surrounding it, because we have got no experimental clue. Therefore, it was not possible to guess any other metric of the space, apart from the usual three-dimensional space of real numbers, in the $20^{\text {th }}$ century.

And therefore, relativistic formulas in the existing version of the STR were derived precisely for such a space. They gave some idea of relativistic effects

$$
\begin{gathered}
m=\frac{m_{0}}{\sqrt{1-(v / c)^{2}}} \\
\Delta t=\Delta t_{0} \sqrt{1-(v / c)^{2}} \\
l=l_{0} \sqrt{1-(v / c)^{2}}
\end{gathered}
$$

where $m_{0}$ is the rest mass of a physical body;

$m$ is the relativistic mass of a moving physical body;

$\Delta t_{0}$ is the rest time of a physical body;

$\Delta t$ is the relativistic time of a moving physical body;

$l_{0}$ is the rest longitudinal length of a physical body;

$I$ is the relativistic longitudinal length of a moving physical body;

$v$ is the velocity of a moving physical body;

$c$ is the speed of light.

Naturally, these formulas and their graphs (Figures 1(a)-(c)) should then be explained in the STR. No wonder, explaining the formulas (1)-(3) in the range $0 \leq v<c$ of the $v$ argument change, the authors of the existing version of the STR found the only three-dimensional space of real numbers known. At the same time they were confronted with an incomprehensible and insuperable circumstance. Relativistic mass $m$, relativistic time $\Delta t$ and relativistic longitudinal length $I$ of a moving physical body calculated by formulas (1)-(3) turned out to

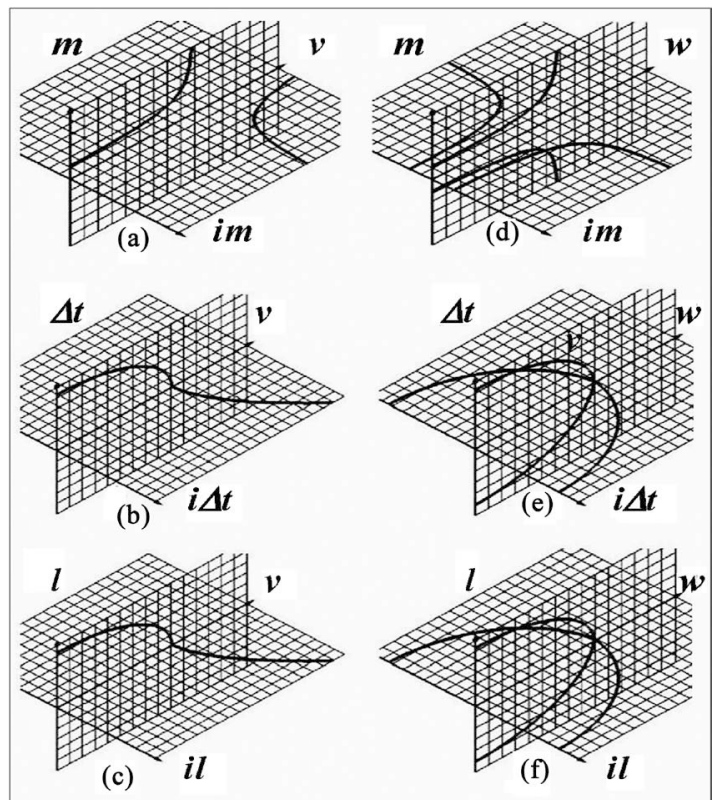

Figure 1. Graphs of functions (1)-(3) and (4)-(6). 
assume imaginary values at $c \leq v$. No one could explain this. And there was not the slightest hope that this could be somehow explained in the near future, since none of great mathematicians and physicists of that time managed to explain physical meaning of imaginary numbers discovered in mathematics four hundred years before creation of the existing version of the STR. Moreover, in the graphs of Figures 1(a)-(c), it can be seen that at $0 \leq v<c$ and $c \leq v<\infty$ the functions $m(v), \Delta t(v)$ and $l(v)$ change in a significantly different way. Therefore, the graphs $m(v), \Delta t(v)$ and $l(v)$ shown in Figures 1(a)-(c) turned out to be incompletely explained.

All this discouraged the creators of the STR. Therefore, further development of the STR ceased for a century at this stage of its creation. In order to protect the available results from scientific ${ }^{2}$ and pseudoscientific ${ }^{3}$ criticism, there was made the only right decision at that time to deny physical reality of imaginary numbers, since it was not proved. For this purpose, the STR introduced the postulate of light speed non-exceedance. It was almost refuted by Nobel Prize received by Pavel Alekseevich Cherenkov, Igor Evgenievich Tamm and Ilya Mikhailovich Frank for discovering and explaining Cherenkov radiation [8] arising when electrically charged particles move through a transparent medium at a speed exceeding the speed of light in the medium. However, later the situation was saved by clarifying that the principle of light speed non-exceedance implied only the speed of light in vacuum.

Nevertheless, a certain natural distrust of the postulated principle of light speed non-exceedance has been preserved. Therefore, attempts to refute it were made. The last was the OPERA experiment. On September 23, 2011 the OPERA collaboration published [9] a sensational report on registration of superluminal neutrinos. However, on March 15, 2012 the ICARUS collaboration published [10] a no less sensational report on refutation of the OPERA experiment. This even created illusion of incontrovertibility of the existing version of the STR.

However, creation of such an illusion was conceivably the true goal of the unsuccessful OPERA experiment, since alternative successful experiments [11]-[29], including those conducted in 2008-2010 [12] [13] [14] [15] [16], i.e. prior to publication of OPERA experiment results, were not taken into account in the

${ }^{2}$ The STR was criticized by Oliver Heaviside, Nikola Tesla, Nobel Prize laureateFriedrich Wilhelm Ostwald, Nobel Prize laureateJoseph John Thomson, Nobel Prize laureate Svante August Arrhenius, Nobel Prize laureatePhilipp Eduard Anton von Lenard, Nobel Prize laureate Alvar Gullstrand, Nobel Prize laureateWilhelm Carl Werner Otto Fritz Franz Wien, Nobel Prize laureateWalther Hermann Nernst, Nobel Prize laureateErnest Rutherford, 1st Baron Rutherford of Nelson, Nobel Prize laureateJohannes Stark, Nobel Prize laureateFrederick Soddy, Nobel Prize laureatePercy Williams Bridgman, Nobel Prize laureateEdwin Mattison McMillan, Nobel Prize laureateHideki Yukawa, Nobel Prize laureate Hannes Ol of Gösta Alfven and many other outstanding scientists.

${ }^{3}$ For example, decisions on banning criticism of the theory of relativity were made three times in the Soviet Union: in 1934, by the resolution of the Central Committee of the All-Union Communist Party (Bolsheviks) on the discussion of relativism; in 1942, by the resolution of the Presidium of the Academy of Sciences of the Soviet Union on the theory of relativity; and in 1964, by the closed decree of the Presidium of the Academy of Sciences of the Soviet Union that forbade all scientific councils, journals and departments to accept, consider, discuss and publish works criticizing the theory of Albert Einstein. 
Internet, although they refuted the principle of light speed non-exceedance and made the OPERA experiment needless. Therefore, assumption that the unsuccessful OPERA experiment was just a promotional event allows us at least somehow explanation of an irrational situation, in which attention was so diligently attracted to the needless and false experiment and successful alternative experiments were ignored.

So, concluding what has been said above, it can be argued that the STR was not created in the $20^{\text {th }}$ century [30] [31], because

- relativistic formulas obtained in its existing version turned out to be incorrect;

- its relativistic formulas were explained incorrectly due to the use of the incorrect principle of light speed non-exceedance;

- conclusions on existence of only our visible Monoverse drawn from its relativistic formulas were incorrect due to the use of the principle of light speed non-exceedance.

Moreover, in the $20^{\text {th }}$ century correct relativistic formulas (10)-(12) could not be obtained and correct version of the STR could not be created, since;

- the principle of physical reality of imaginary numbers refuting experimentally the postulated (i.e. being an unproven assumption) principle of light speed non-exceedance was published only in the $21^{\text {st }}$ century;

- experimental data whose mathematical analysis discerned the quaternion structure of the hidden Multiverse were obtained by WMAP and Planck spacecraft only in the $21^{\text {st }}$ century;

- explanation of the way how astronomical observations of constellations of the starry sky in portals can experimentally prove the existence of invisible universes was published only in 2019.

An alternative version of the STR free of the shortcomings of its existing version is presented below in the article.

\section{The Logic of Reasoning That Led to Creation of the Alternative Version of the STR}

Creation of the alternative version of the STR differed from its generally recognized version. Its basic premise was experimental proof of the principle of physical reality of concrete imaginary numbers, so indisputably refuted by the light speed non-exceedance postulate available in the existing version of the STR. Further, the analysis of WMAP and Planck experimental data using the principle of physical reality of imaginary numbers allowed determining ${ }^{4}$ the metric of space we live in and refining the structure of our Multiverse. Our Multiverse turned out to consist of twenty to twenty two mutually invisible parallel universes and have a quaternion structure in six-dimensional space. Therefore, it is called the hidden Multiverse. Notably, existence of invisible parallel universes can be confirmed by astronomical observations made from portals available on

${ }^{4}$ And do not guess it with the postulates, as in the existing version of the STR. 
Earth.

Details are given below.

\subsection{Proofs of Physical Reality of Concrete Imaginary Numbers}

In contrast to existing version of the STR, its alternative version primarily proves the principle of physical reality ${ }^{5}$ of concrete imaginary numbers ${ }^{6}$. And even three experimental proofs have been proposed:

- The first one has been obtained in analysis of oscillatory transient processes. Hence, it follows that there would be no tsunami, church bells would not ring and even children's swing wouldn't sway after being pushed by parents [16] [17] [20] [21] [27] [28] [29], if the statement of physical unreality of imaginary numbers contained in the existing version of the STR were true.

- The second one has been obtained in analysis of oscillatory resonant processes. Hence, it follows that there would be no television and telecommunication, radiolocation and radio navigation, as well as many other exact sciences [12] [13] [14] [15] [19] [20] [27] [28] [29], if the statement of physical unreality of imaginary numbers contained in the existing version of the STR were true.

- The third one has been obtained in analysis of forced oscillatory processes in alternating current electric circuits. Hence, it follows that Ohm's law wouldn't exist for alternating current electric circuits [22] [23] [24] [25] [26], [28] [29], if the statement of physical unreality of imaginary numbers contained in the existing version of the STR were true.

All these experimental proofs, unlike the extremely complex and expensive unique OPERA experiment, can be verified in any radio engineering laboratory. Now they are daily confirmed by practical activities of millions of electric and radio engineers. Consequently, they are guaranteedly faithful and absolutely conclusive. Nevertheless, the physical reality of imaginary numbers still has to be proved, even contrary to Ohm's law [23] [24], which indicates the imperfection of modern physical education, since in SRT the principle of not exceeding the speed of light is still assumed to be true $\mathrm{e}^{7}$.

Therefore, it can be argued that physically real imaginary numbers correspond to an invisible world unknown to us, which remains to be known to the science of the future. The utmost importance of imaginary numbers in the science was noted by Sir Roger Penrose: "The very system of complex numbers has a profound and timeless reality which goes beyond the mental constructions of any particular mathematician... They were put there neither by Cardano, nor by

${ }^{5}$ Which in the current version of SRT is denied by the postulated principle of non-exceeding the speed of light.

${ }^{6}$ Naturally, it makes sense to talk about physical reality of imaginary, complex and hypercomplex numbers, as well as real numbers, only when it comes to concrete numbers provided with references to units used to measure parameters of corresponding physical objects and processes.

${ }^{7}$ The situation when postulates are sometimes used for lack of experiments is acceptable in science. But when the postulates disprove experiments, such a situation goes beyond common sense and is unacceptable in science. 
Bombelly, nor Wallis, nor Coates, nor Euler, nor Wessel, nor Gauss, despite the undoubted farsightedness of these, and other, great mathematicians; such magic was inherent in the very structure that they gradually uncovered".

From the alternative version of the SRT, the principle of not exceeding the speed of light is therefore excluded. And the principle of the physical reality of imaginary numbers, on the contrary, now needs to be recognized as a general scientific one, and in accordance with this principle all theories and hypotheses should be corrected.

Let us show how this can be done, for example, in the STR.

\subsection{Relativistic Formulas of the Alternative Version of the STR}

Relativistic formulas (1)-(3) of the existing version of the STR are corrected in the alternative version of the STR as follows. Since the principle of physical reality of imaginary numbers disproves the postulate of not exceeding the speed of light, formulas (1)-(3) might be explainable at argument values $v$ that are both lesser and greater than $c$. However, since they still defy explanation at $c \leq v$, formulas (1)-(3) have to be recognized as incorrect. And for the corrected relativistic formulas to be explainable, graphs of functions $m(v), \Delta t(v)$ and $l(v)$ should be comparable at argument values $v$ that are both lesser and greater than c, i.e. should be as shown in Figures 1(d)-(f). They correspond to the following formulas

$$
\begin{gathered}
m=\frac{m_{0} i^{q}}{\sqrt{1-(v / c-q)^{2}}}=\frac{m_{0} i^{q}}{\sqrt{1-(w / c)^{2}}} \\
\Delta t=\Delta t_{0} i^{q} \sqrt{1-(v / c-q)^{2}}=\Delta t_{0} i^{q} \sqrt{1-(w / c)^{2}} \\
l=l_{0} i^{q} \sqrt{1-(v / c-q)^{2}}=l_{0} i^{q} \sqrt{1-(w / c)^{2}}
\end{gathered}
$$

where $q=\lfloor v / c\rfloor$ is the "floor" function of argument $v / c$;

$w=v-q c$ is the local velocity for each universe, which can take values only in the range $0 \leq w<c$;

$V$ is the velocity measured from our universe;

$c$ is the speed of light.

Albert Einstein did not exclude such correction of the STR in future. He wrote: "There is no single idea, which I would be sure that it will stand the test of time".

\subsection{Structure of the Hidden Multiverse}

It follows from formulas (4)-(6) that there is a Multiverse [32]-[39], rather than a Monoverse, as stated in the existing version of the STR. And different quantities $q$ in formulas (4)-(6) correspond to different physically real universes. The quantity $q=0$ in formulas (4)-(6) corresponds (as $i^{0}=1$ ) to our universe, and the quantity $q=1$ corresponds (as $i^{1}=i$ ) to the adjacent universe, in which $c \leq v<2 c$ and which is therefore invisible from our universe, since it is located 
beyond the event horizon. Consequently, this is the universe containing tachyons that do not violate the principle of causality [40]-[45].

Let us, therefore, call it a tachyon universe. For the same reasons our universe shall be referred to as a tardyon universe. Subsequently:

- the quantity $q=2$ in formulas (4)-(6) corresponds to the invisible (as $2 c \leq v<3 c$ for it) tardyon antiverse ${ }^{8}$ (as $i^{2}=-1$ );

- the quantity $q=3$ in formulas (4)-(6) corresponds to the invisible (as $3 c \leq v<4 c$ for it) tachyon antiverse (as $i^{3}=-i$ );

- the quantity $q=4$ in formulas (4)-(6) corresponds to the invisible (as $4 c \leq v<5 c$ for it) another tardyon universe (as $i^{4}=1$ );

- the quantity $q=5$ in formulas (4)-(6) corresponds to the invisible (as $5 c \leq v<6 c$ for it) another tachyon universe (as $i^{5}=i$ ) etc.

All universes in this Multiverse are mutually invisible and therefore it shall be called the hidden Multiverse. Distribution of physical contents in this hidden Multiverse is described by the function $f_{q}(x, y, z)+i q$, where $x, y, z$ are the coordinates of physical contents in a corresponding parallel universe, and $q$ is the coordinate of this universe in the fourth spatial dimension.

Moreover, invisible parallel ${ }^{9}$ universes do not actually stand still in such a four-dimensional space ${ }^{10}$, but continuously drift and very often slightly penetrate into each other in many spots, generating transition zones ${ }^{11}$. Such zones are usually called portals ${ }^{12}$ or star gates [46] [47] [48] [49] [50]. Figure 2 shows an example of structure of such a hidden Multiverse, which, as can be seen, is helical. Numerous bidirectional portals in the structure are indicated by single two-sided arrows.

\subsection{Explanation of the Phenomenon of Dark Matter and Dark Energy}

The WMAP [51] and Planck [52] spacecraft were launched into space to solve problems that would seem to have nothing to do with the contents of this article. They did not aim to promote the creation of an alternative version of the STR instead of its existing version, which is still considered unshakably true. They were created to study relic radiation produced by the Big Bang.

They also allowed to determine that the universe is composed of:

- $4.6 \%$ baryonic matter according to WMAP data (or $4.9 \%$ according to Planck data);

- $22.4 \%$ dark matter according to WMAP data (or 26.8\% according to Planck data);

- $73.0 \%$ dark energy according to WMAP data (or $68.3 \%$ according to Planck data).

${ }^{8}$ Which contains antimatter, like other antiverses. And it does not annihilate with matter, because tardyon and tachyon universes and antiverses alternate in the hidden Multiverse.

${ }^{9}$ Since they never intersect despite their infinity.

${ }^{10}$ Non-Minkowski space.

${ }^{11}$ In which the quantity $q$ varies by one from one integer value to another, corresponding to adjacent parallel universes.

${ }^{12}$ Which have nothing to do with 'wormholes' in the general theory of relativity. 


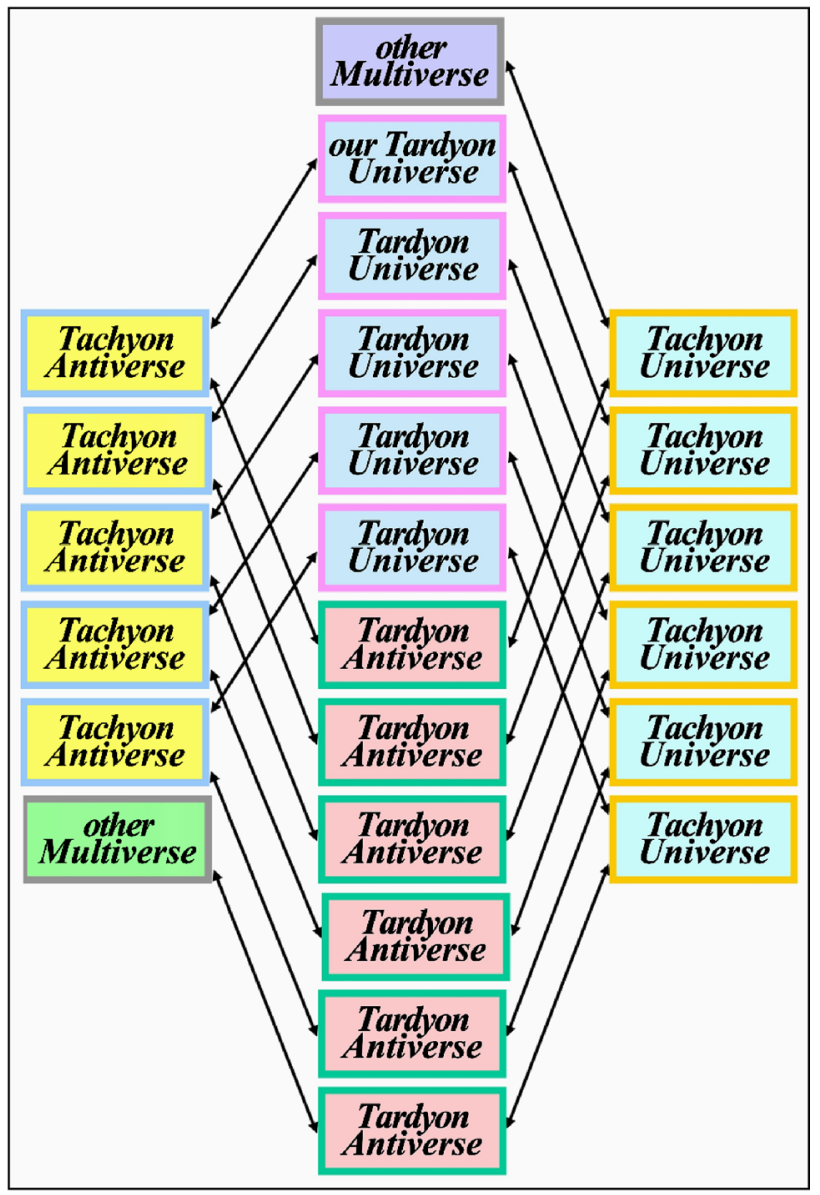

Figure 2. Probable structure of the hidden Multiverse corresponding to the principle of physical reality of complex numbers.

That is, the universe (more precisely, the hidden Multiverse) turned out to be more than $95 \%$ composed of dark matter and dark energy. Dark matter was discovered by Jan Hendrik Oort [53] and Fritz Zwikky [54] in 1932-33. Dark energy was discovered by Saul Perlmutter [55], Brian Schmidt [56] and Adam Riess [57] in 1998-1999. They were awarded the Nobel Prize for this discovery. Stressing the importance of the discoveries, the Nobel Prize laureate Adam Riess wrote: "Humanity is on the verge of a new physics of the Universe. Whether we want it or not, we will have to accept it". The new physics of the Universe is concerned below.

Despite extremely diligent efforts to study dark matter and dark energy [58]-[64], they still seem completely incomprehensible in the existing version of the STR. Therefore, they were called dark. The famous astrophysicist and professor MichioKaku argued: "Of course, a whole bunch of Nobel Prizes is waiting for the scientists who can reveal the secrets of the 'dark energy' and 'dark matter'."

However, it is easy to see that the phenomenon of dark matter and dark energy is incomprehensible only because its explanation has so far been sought exclusively within the framework of the Monoverse hypothesis corresponding to 
the existing version of the STR.

In this regard, it would not be out of place to take into account the opinion of Albert Einstein: "Insanity: doing the same thing over and over again and expecting different results."

And when using the hypothesis of the hidden Multiverse, the phenomenon of dark matter and dark energy turned out to be quite explainable [20] [25] [26] [29] [35] [36] [37] [45] [65]-[70]:

- dark matter and dark energy are actually a kind of image (gravitational rather than optical or even electromagnetic), something like a shadow, evoked by existence of invisible parallel universes;

- therefore, any physical content, such as molecules, atoms or subatomic particles, will never be found in dark matter and dark energy;

- the dark matter phenomenon is evoked by invisible parallel universes adjacent to our visible universe, whereas the dark energy phenomenon is evoked by other invisible parallel universes of the hidden Multiverse.

Consequently, believing that mass-energy of invisible parallel universes has been substantially averaged over billions of years due to existence of portals, their mass-energy can be accurately assumed to be equal. Therefore, we deduce the following:

- The total number of invisible parallel universes in the hidden Multiverse is $100 \% / 4.6 \%=21.7$ universes according to WMAP data and $100 \% / 4.9 \%=20.4$ universes according to Planck data, i.e. 20 ... 22 universes;

- The number of invisible parallel universes evoking the phenomenon of dark matter is $22.4 \% / 4.6 \%=4.9$ universes according to WMAP data and $26.8 \% / 4.9 \%=5.5$ universes according to Planck data, i.e. 5 .. 6 universes;

- The number of invisible parallel universes evoking the phenomenon of dark energy is $73.0 \% / 4.6 \%=15.9$ universes according to WMAP data and $68.3 \% / 4.9 \%=13.9$ universes according to Planck data, i.e. $14 \ldots 16$ universes.

\subsection{Relativistic Formulas of the Alternative Version of the STR (Continued)}

Thus, although the WMAP and Planck spacecraft were sent to space for another purpose, the data they received allowed clarifying the structure of the hidden Multiverse and thereby provided experimental support for creation of the alternative version of the STR.

But it is easy to see that results of mathematical processing of WMAP and Planck spacecraft data are inconsistent with formulas (4)-(6) and the structure of the hidden Multiverse shown in Figure 2. In Figure 2 only one tachyon universe and one tachyon antiverse are actually adjacent to each tardyon universe and antiverse, rather than five or six tachyon universes and antiverses as according to the calculations. The thing is that there is no space for placing five or six invisible parallel universes near each tardyon universe or antiverse in the structural 
diagram given in Figure 2. Consequently, there are three extra dimensions $q, r, s$ in our hidden Multiverse, rather than one $q$. In this regard, formulas (4)-(6) corresponding to the principle of physical reality of complex numbers should be corrected in accordance with the principle of physical reality of quaternions [71] containing three imaginary units $i_{1}, i_{2}, i_{3}$ interconnected by the relations

$$
\begin{gathered}
i_{1}^{2}=i_{2}^{2}=i_{3}^{2}=1 \\
i_{1} i_{2} i_{3}=i_{2} i_{3} i_{1}=i_{3} i_{1} i_{2}=-1 \\
i_{1} i_{3} i_{2}=i_{2} i_{1} i_{3}=i_{3} i_{2} i_{1}=1
\end{gathered}
$$

The corrected formulas are written as follows

$$
\begin{aligned}
m= & \frac{m_{0}\left(i_{1}\right)^{q}\left(i_{2}\right)^{r}\left(i_{3}\right)^{s}}{\sqrt{1-[v / c-(q+r+s)]^{2}}}=\frac{m_{0}\left(i_{1}\right)^{q}\left(i_{2}\right)^{r}\left(i_{3}\right)^{s}}{\sqrt{1-(w / c)^{2}}} \\
\Delta t & =\Delta t_{0}\left(i_{1}\right)^{q}\left(i_{2}\right)^{r}\left(i_{3}\right)^{s} \sqrt{1-[v / c-(q+r+s)]^{2}} \\
& =\Delta t_{0}\left(i_{1}\right)^{q}\left(i_{2}\right)^{r}\left(i_{3}\right)^{s} \sqrt{1-(w / c)^{2}} \\
l & =l_{0}\left(i_{1}\right)^{q}\left(i_{2}\right)^{r}\left(i_{3}\right)^{s} \sqrt{1-[v / c-(q+r+s)]^{2}} \\
& =l_{0}\left(i_{1}\right)^{q}\left(i_{2}\right)^{r}\left(i_{3}\right)^{s} \sqrt{1-(w / c)^{2}}
\end{aligned}
$$

where $q$ is the total number of parallel universes, penetration into which is made through portals, corresponding to the imaginary unit $i_{1}$, with increasing distance from our tardyon universe;

$r$ is the total number of parallel universes, penetration into which is made through portals, corresponding to the imaginary unit $i_{2}$, with increasing distance from our tardyon universe;

$s$ is the total number of parallel universes, penetration into which is made through portals, corresponding to the imaginary unit $i_{3}$, with increasing distance from our tardyon universe;

$v$ is the velocity measured from our tardyon universe;

$c$ is the speed of light;

$w=v-(q+r+s) c$ is the local velocity for corresponding universe, which can take values only in the range.

And it is quite obvious that the above given WMAP and Planck research data that allowed deriving relativistic formulas (10)-(12) for the alternative version of the STR could be guessed by no postulates. Therefore, the Nobel Prize laureate Stephen Weinberg clearly remarked on the theories created using the postulates: "Scientific theories cannot be deduced by purely mathematical reasoning". In other words, no true physical theory can be created without experimental clues.

\subsection{Structure of the Hidden Multiverse (Continued)}

As can be seen, the results obtained are inconsistent with perceptions generally accepted in relativistic physics and astrophysics. However, Sir Isaac Newton ar- 
gued: "No great discovery was ever made without a bold guess". The same opinion was held by the Nobel laureate Niels Henrik David Bohr who said his catch phrase: "There is no doubt we have faced a mad theory. But the question is this. Is it really crazy enough to be right?"

The hidden Multiverse corresponding to formulas (10)-(12) can have the structure shown in Figure 3. The structure looks like an open helical ring the ends of which are connected to two other Multiverses. Besides, the hidden Multiverse can be connected to other Multiverses in another way. As can be seen, the quaternion structure [72] differs from the structure shown in Figure 2 by containing three tachyon universes $i_{1}, i_{2}, i_{3}$ and three tachyon antiverses $i_{1}, i_{2}, i_{3}$, which provides three necessary extra dimensions. Thus, six-dimensional space of the hidden Multiverse (Figure 4) has actually three extra dimensions $q, r, s$ containing parallel universes, and three dimensions $x, y, z$ containing physical contents of each of these universes. That is, space of such a hidden Multiverse is described by the formula $f_{q, r . s}(x, y, z)+i_{1} q+i_{2} r+i_{3} s$, where the function $f_{q, r, s}(x, y, z)$ describes distribution in coordinates $x, y, z$ of physical content in the corresponding parallel universe having the coordinates $q, r, s$.

A member of the US National Academy of Sciences Lisa Randall wrote in this regard: "We can be living in a three-dimensional space sinkhole in a higher-dimensional universe". Apparently, her assumption was justified.

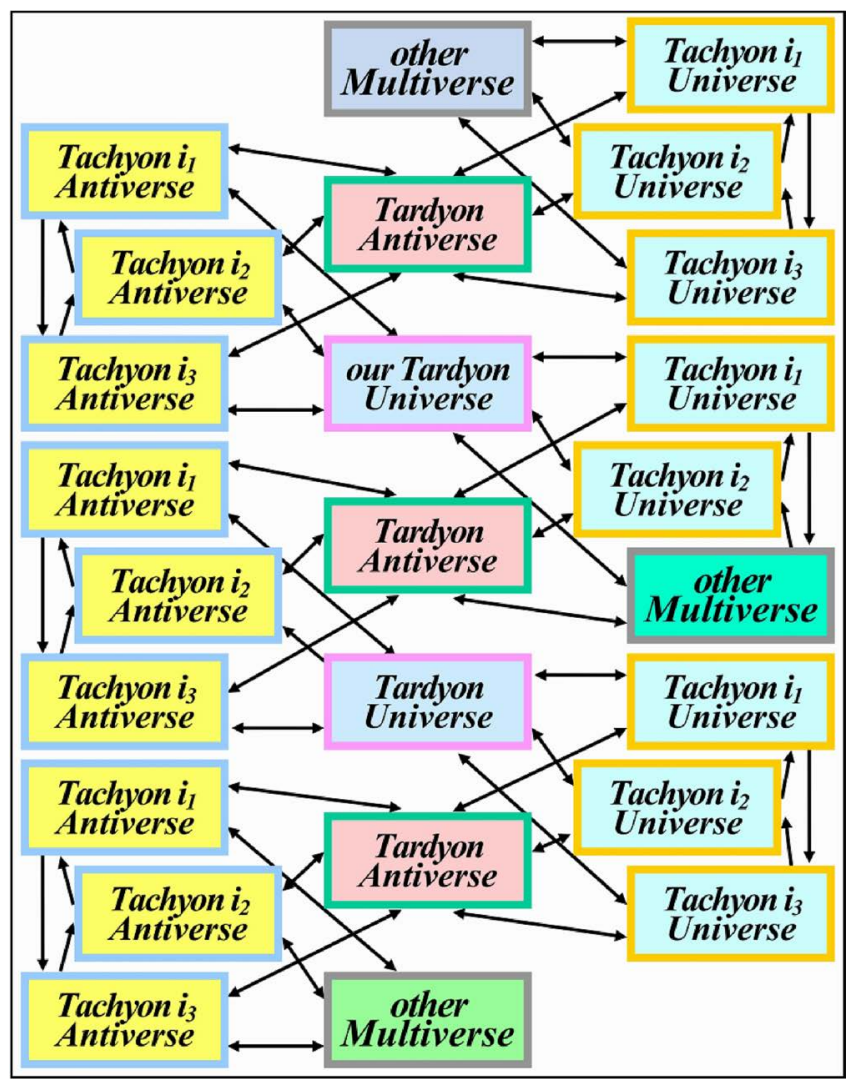

Figure 3. Example of structure of the hidden Multiverse corresponding to the principle of physical reality of quaternions. 


\subsection{Explanation of the Phenomenon of Dark Space}

Previous Figure 3 shows that our hidden Multiverse is united with other Multiverses through the corresponding portals and forms the Hyperverse together with them. Therefore, it can be argued that other invisible Multiverses of the Hyperverse except our hidden Multiverse form dark space [29] [73]. Herewith, invisible parallel universes of the Multiverses of dark space may presumably be connected to our visible tardyon universe through the corresponding portals, as in Figure 5(b), or may not, as shown in Figure 5(a).

However, availability or lack of such connections cannot be ascertained by astrophysical studies of the WMAP and Planck spacecraft, since otherwise the registered universes would have been classified as universes of dark matter or dark energy.

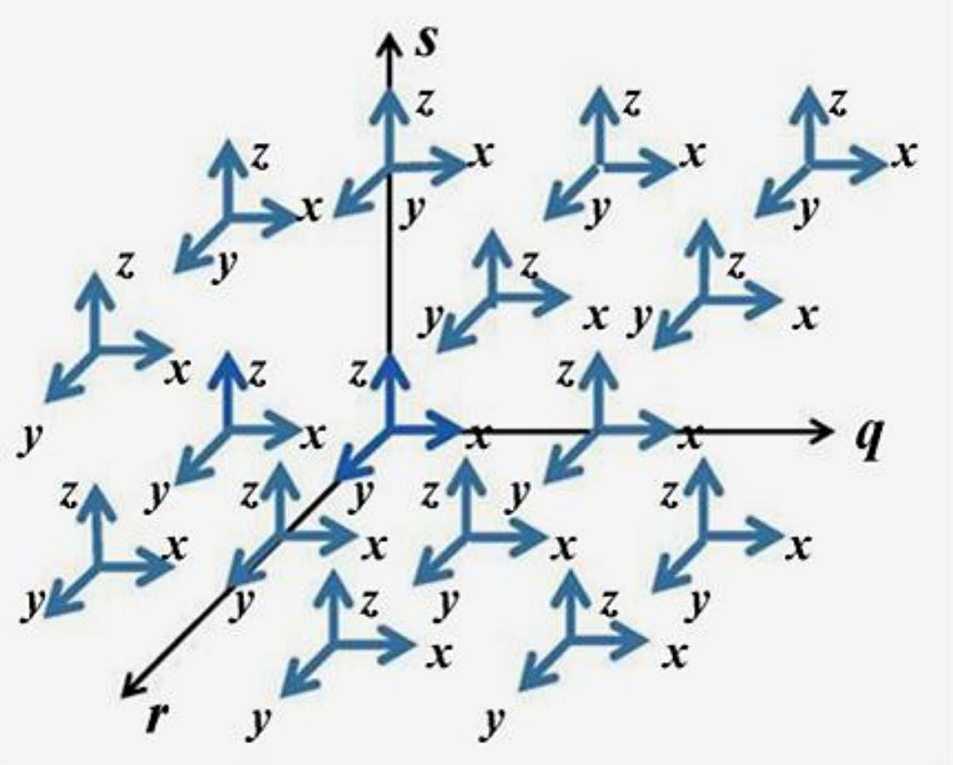

Figure 4. Six-dimensional space of the hidden Multiverse.

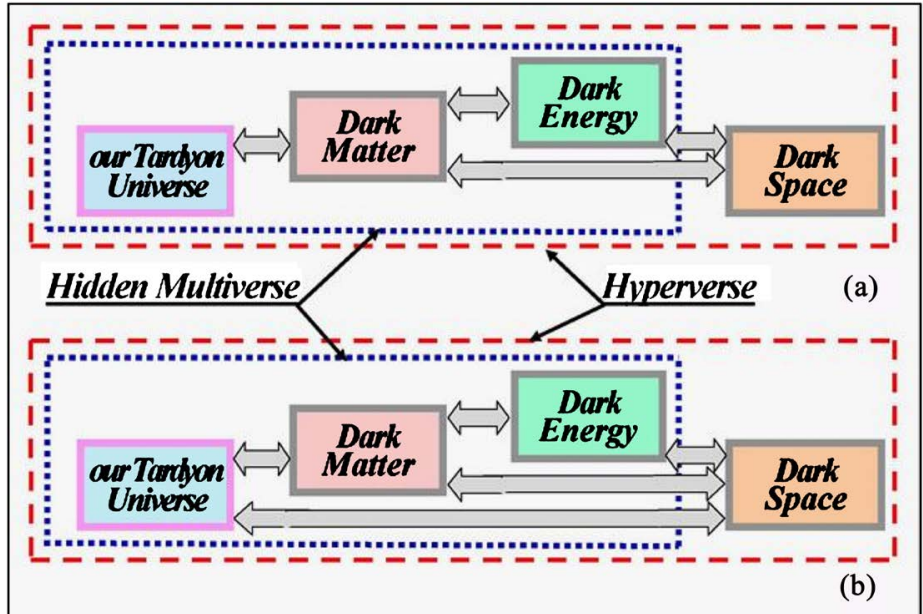

Figure 5. Structure of the Hyperverse. 
So, how to make sure of existence of dark space invisible universes? And can this be verified anyway? Apparently, yes. Even in two different ways. First way is astrophysical research of portals. This can be unsafe for people. But for this purpose robotic mobile systems similar to WMAP and Planck spacecraft can be created. Second way is astronomical observations of starry sky constellations made from portals. They are described below.

\subsection{Experimental Proof of the Alternative Version of the STR}

Thus, we can state that two hypotheses of dark matter and dark energy have been proposed by now. And both are unusual enough to claim to be true in accordance with the criteria of Isaac Newton and Niels Bohr.

The first hypothesis-corresponding to the existing version of SRT-is better known. It suggests that explanation of dark matter and dark energy should be sought in the microcosm. Therefore, it is sought by research at the Large Hadron Collider.

The second hypothesis-corresponding to the alternative version of SRT-is almost unknown set forth in the article. It suggests that explanation of dark matter and dark energy should be sought in the macrocosm. And it is based on the existence of invisible parallel universes of our hidden Multiverse.

There is a good chance that a third hypothesis may be proposed and that it may be even more unusual and appear to be the truest.

But for the time being all of them are just hypotheses. And only an experiment can show which of them will ultimately become a theory [74]. As concerns science, only experiments decide which hypotheses are true or wrong ${ }^{13}$. Therefore, supporters of the first hypothesis search for such a decisive argument at the Large Hadron Collider so persistently. The second hypothesis will also be recognized as true only if it gets experimental confirmation.

It can get the confirmation in the course of astronomical observations of starry sky constellations made from portals [75]. Let's give a comparison to make this idea more clear. The room of our house we are in now is our visible world, whereas the next room is invisible world. However, we can make certain of its existence by TV sounds heard there from. We can see it partially, coming closer to the door and sticking head therein. We can even see the next room entirely, entering it through the door. In this case the room we were in before would become invisible to us.

Similarly, we can partially see the invisible universe, entering the portal. And the further we are, the more we see. The next invisible universe can be seen entirely after entering it through the portal. Thus the last visible universe would become invisible to us. The snag is that portals are invisible too. It is not easy to

${ }^{13}$ In the Thirty Years' War Cardinal Richelieu, driven by the same reasons, ordered to inscribe upon cannons the following text: "Ultima ratio regum". And the last argument of scientists is experiments. Only by experiments can the postulates be confirmed or disproved. But the experiments cannot be either confirmed or disproved by postulates. 
get into the next universe through them. Much easier is to get lost in portals and not to return to our world.

Therefore, deepening into portals requires special portal orientation equipment (just as a marine compass used by sailors). Creating the equipment, it should be taken into account that all radio signals fade down with your dipping into the portal and gradually disappear once you are in the adjacent universe.

However, people should not put themselves in danger for such research. Instead, robotic systems can be sent to the portals. They are much easier to create than WMAP and Planck spacecraft.

Moreover, one can see the edge of the adjacent universe even with a shallow dipping into portals. This can be verified by observation of the changed constellation pattern similar to that made by Sir Arthur Stanley Eddington in 1919 [76].

\section{Conclusions}

Thus, the relativistic formulas obtained in the existing version of the SRT were incorrect due to the lack of experimental support, their conclusion was not completed, they were incorrectly explained, and the conclusions drawn from them about the physical unreality of imaginary numbers and the existence of only our visible Mono-Universe in nature were also incorrect. In other words, the existing version of SRT is incorrect.

Nevertheless, the existing version of STR is a great scientific achievement of Albert Einstein, who created the relativistic physics. Without creating an existing version of the SRT, it would be impossible to create its alternative version.

But in the alternative version of STR, relativistic formulas were created using experimental data obtained already in the $21^{\text {st }}$ century ${ }^{14}$ :

- experimentally proven principle of physical reality of imaginary numbers refuting the postulated principle of light speed non-exceedance;

- WMAP and Planck data whose mathematical processing allowed to determine the structure of the hidden Multiverse.

The new relativistic formulas using the principle of physical reality of imaginary numbers are fully explainable. It follows from them that in reality there is not a Monoverse, as stated in all physics textbooks, but a multitude of mutually invisible parallel universes, which together form a hidden Multiverse. And from the data obtained by the WMAP and Planck spacecraft, it follows that the hidden Multiverse exists in six-dimensional space and has a quaternionic structure. In addition, it follows from these data that the invisible Multiverses exist outside the hidden Multiverse, with which they form the Hyperverse.

Such a hypothesis of the hidden Multiverse and Hyperverse made it possible to explain the phenomenon of dark matter and dark energy by a peculiar gravitational shadow of other invisible universes of our visible universe. Such an explanation of the phenomenon of dark matter and dark energy also made it possible to understand why in studies at the Large Hadron Collider it was not possi-

\footnotetext{
${ }^{14}$ And it is possible that as a result of new experimental data obtained in the future, the relativistic formulas presented in this article will be corrected again, possibly even repeatedly.
} 
ble to detect material carriers of this phenomenon. And the invisible universes, located beyond the borders of the hidden Multiverse, generated the phenomenon of dark space.

The hypothesis of the hidden Multiverse and Hyperuniverse set forth in the alternative version of SRT also explains where antimatter is located and why it does not annihilate with matter, as well as where are tachyons, which do not violate the principle of causality. These explanations are simple and straightforward. Antimatter is found in numerous antiverses, since there are many universes. And tachyons that do not violate the principle of causality are in numerous tachyon universes and anti-universes.

The invisible parallel universes of the hidden Multiverse and Hyperuniverse in six-dimensional space naturally drift relative to each other. Therefore, in many places, neighboring invisible universes are slightly immersed in each other, forming portals through which exchange of their material contents between these universes is possible. On our planet, at least some of the existing anomalous zones are such portals. And from these portals, even with a shallow penetration in them, one can see the edge of the starry sky in neighboring universes with constellations other than we see outside the portals. Thus, such astronomical observations can prove the existence of invisible universes. And for the entire history of the existence of astronomy, there was no more interesting and more important task in it.

\section{Conflicts of Interest}

The author declares no conflicts of interest regarding the publication of this paper.

\section{References}

[1] Einstein, A. (1920) Relativity: The Special and General Theory. H. Holt and Company, New York.

[2] Bohm, D. (2006) The Special Theory of Relativity. Routledge, London.

[3] Hawking, S.W. and Penrose, R. (2010) The Nature of Space and Time. Princeton University Press, Princeton, NJ. https://doi.org/10.1515/9781400834747

[4] Larmor, J.J. (1897) Philosophical Transactions of the Royal Society A: Mathematical, Physical and Engineering Sciences, 190, 205-300.

[5] Lorentz, H.A. (1899) Proceeding of the Royal Netherlands Academy of Arts and Science, 1, 427-442.

[6] Poincaré, H. (1905) Comptes Rendus, 140, 1504-1508.

[7] Einstein, A. (1905) Annalen der Physik, 17, 891-921. https://doi.org/10.1002/andp.19053221004

[8] Čerenkov, P.A. (1937) Physical Review, 52, 378-379. https://doi.org/10.1103/PhysRev.52.378

[9] Adam, T., Agafonova, N., Aleksandrov, A., et al. (2012) Measurement of the Neutrino Velocity with the OPERA Detector in the CNGS Beam. arXiv:1109.4897v4.

[10] Antonello, M., Baibussinov, B., Boffelli, F., et al. (2012) Precision Measurement of 
the Neutrino Velocity with the ICARUS Detector in the CNGS Beam. arXiv:1208.2629v2.

[11] Lyahov, V.V. and Nechshadim, V.M. (2001) Complex Numbers and Physical Reality. arXiv:physics/0102047v2. (In Russian)

[12] Antonov, A.A. (2008) European Journal of Scientific Research, 21, 627-641.

[13] Antonov, A.A. (2009) European Journal of Scientific Research, 28, 193-204.

[14] Antonov, A.A. (2010) American Journal of Scientific and Industrial Research, 1, 342-349. https://doi.org/10.5251/ajsir.2010.1.2.342.349

[15] Antonov, A.A. (2010) International Journal of Pure and Applied Sciences and Technology, 1, 1-12.

[16] Antonov, A.A. (2010) General Mathematics Notes, 1, 11-16.

[17] Antonov, A.A. (2013) International Journal of Management, IT and Engineering, 3, 219-230.

[18] Spenkov, G.P. (2013) Encyclopedia of Russian thought, 20, 69-80. (In Russian) http://spenkov.janmax.com/ImaginUnitRus.pdf

[19] Antonov, A.A. (2014) American Journal of Scientific and Industrial Research, 5, 40-52.

[20] Antonov, A.A. (2015) Journal of Russian Physical and Chemical Society, 87, 328-355. (In Russian)

[21] Antonov, A.A. (2015) General Mathematics Notes, 31, 34-53. http://www.emis.de/journals/GMN/yahoo site admin/assets/docs/4 GMN-9212-V 31N2.1293701.pdf

[22] Antonov, A.A. (2016) PONTE, 72, 131-142. https://doi.org/10.21506/j.ponte.2016.9.22

[23] Antonov, A.A. (2015) American Journal of Electrical and Electronics Engineering, 3, 124-129. https://doi.org/10.1016/j.proeng.2015.12.088

[24] Antonov, A.A. (2016) Journal of Modern Physics, 7, 2299-2313. https://doi.org/10.4236/jmp.2016.716198

[25] Antonov, A.A. (2015) Global Journal of Physics, 2, 145-149. http://gpcpublishing.com/index.php?journal=gjp\&page=article\&op=view\&path\%5 B\%5D=294\&path\%5B\%5D=pdf 14

[26] Antonov, A.A. (2016) International Review of Physics, 10, 31-35. https://www.praiseworthyprize.org/jsm/index.php?journal=irephy\&page=article\&o $\mathrm{p}=$ view\&path\%5B\%5D=18615

[27] Antonov, A.A. (2016) General Mathematics Notes, 35, 40-63.

[28] Antonov, A.A. (2017) Norwegian Journal of Development of the International Science, 6, 50-63. http://www.njd-iscience.com

[29] Antonov, A.A. (2018) Natural Science, 10, 11-30. https://doi.org/10.4236/ns.2018.101002

[30] Antonov, A.A. (2019) Journal of Russian Physical-Chemical Society, 91, 57-94. (In Russian) http://www.rusphysics.ru/files/Antonov.91-1.pdf

[31] Antonov, A.A. (2019) Scientific Discussion (Praha, Czech Republic), 38, 27-37. http://scientific-discussion.com/ru/archive/

[32] Antonov, A.A. (2011) British Journal of Science, 2, 51-60.

[33] Antonov, A.A. (2012) Encyclopedia of Russian Thought: Reports to Russian Physical Society, 16, 3-20. (In Russian) 
[34] Antonov, A.A. (2012) International Journal of Pure and Applied Sciences and Technology, 12, 43-56.

[35] Antonov, A.A. (2015) International Journal of Advanced Research in Physical Science, 2, 25-32.

[36] Antonov, A.A. (2015) American Journal of Modern Physics, 4, 1-9.

[37] Antonov, A.A. (2015) International Journal of Physics, 3, 84-87.

[38] Antonov, A.A. (2015) Cosmology, 19, 40-61.

[39] Antonov, A.A. (2017) Natural Science, 9, 43-62. https://doi.org/10.4236/ns.2017.93005

[40] Feinberg G. (1967) Physical Review, 159, 1089-1105. https://doi.org/10.1103/PhysRev.159.1089

[41] Bilaniuk, O.-M.P. and Sudarshan, E.C.G. (1969) Physics Today, 22, 43-51. https://doi.org/10.1063/1.3035574

[42] Recami, E., Fontana, F. and Garavaglia, R. (2000) International Journal of Modern Physics A, 15, 2793-2812. https://doi.org/10.1142/S0217751X00001403

[43] Tanaka, S. (1960) Progress of Theoretical Physics (Kyoto), 24, 171-200. https://doi.org/10.1143/PTP.24.171

[44] Terletsky, Y.P. (1966) Paradoxes of the Relativity Theory. Nauka Publishing, Moscow. (In Russian)

[45] Hill, J.M. and Cox, B.J. (2012) Proceeding of Royal Society A.: Mathematical, Physical and Engineering sciences, 468, 4174-4192.

https://doi.org/10.1098/rspa.2012.0340

[46] Antonov, A.A. (2015) Optics, 4, 43-47. https://doi.org/10.1016/j.aml.2015.03.001

[47] Antonov, A.A. (2016) Journal of Modern Physics, 7, 1933-1943. https://doi.org/10.4236/jmp.2016.714170

[48] Antonov, A.A. (2015) Where to Look for Alien Civilisations. Commentaries: Stephen Hawking's Aliens. The Search for Intelligent Extraterrestrial Life. Project Break-through Liste. http://cosmology.com/Aliens1.html

[49] Antonov, A.A. (2016) How Portals of the Invisible Multiverse Operate. SciencePG Frontiers. http://www.sciencepublishinggroup.com/news/sciencepgfrontiersinfo?articleid=147

[50] Antonov, A.A. (2016) Philosophy and Cosmology, 6, 11-27.

[51] Hinshaw, G., Larson, D., Komatsu, E., et al. (2013) Nine Year Wilkinson Anisotropy Probe (WMAP) Observations: Cosmological Parameter Results. arXiv: 1213.5226.

[52] Adam, R., Ade, P.A.R., Aghanim, N., et al. (2015) Plank 2015 Results. 1. Overview of Products and Scientific Results. arXiv:1502.01582v2.

[53] Oort, J.H. (1932) Bulletin of the Astronomical Institutes of the Netherlands, 6, 249-287.

[54] Zwicky, F. (1933) Helvetica Physica Acta, 6, 110-127.

[55] Perlmutter, S. (2012) Reviews of Modern Physics, 84, 1127-1149. https://doi.org/10.1103/RevModPhys.84.1127

[56] Schmidt, B.P. (2012) Reviews of Modern Physics, 84, 1151-1163. https://doi.org/10.1103/RevModPhys.84.1151

[57] Riess, A.G. (2012) Reviews of Modern Physics, 84, 1165-1175. https://doi.org/10.1103/RevModPhys.84.1165 
[58] Freeman, K. and McNamara, G. (2006) In Search of Dark Matter. Springer, Berlin.

[59] Nicolson, I. (2007) Dark Side of the Universe: Dark Matter, Dark Energy, and the Fate of the Cosmos. Johns Hopkins University Press, Baltimore, MD.

[60] Ruiz-Lapuente, P. (2010) Dark Energy: Observational and Theoretical Approaches. Cambridge University Press, Cambridge. https://doi.org/10.1017/CBO9781139193627

[61] Amendola, L. and Tsujikawa, S. (2010) Dark Energy: Theory and Observations. Cambridge University Press, Cambridge. https://doi.org/10.1017/CBO9780511750823

[62] Bertone, G. (2013) Particle Dark Matter: Observations, Models and Searches. Cambridge University Press, Cambridge.

[63] Sanders, R.H. (2014) The Dark Matter Problem: A Historical Perspective. Cambridge University Press, Cambridge.

[64] DeGrasse, T.N. (2017) Astrophysics for People in a Hurry. W. W. Norton \& Company, London.

[65] Antonov, A.A. (2015) American Journal of Modern Physics, 4, 180-188. https://doi.org/10.11648/j.ajmp.20150404.14

[66] Antonov, A.A. (2016) Frontiers of Astronomy, Astrophysics and Cosmology, 2, 1-9.

[67] Antonov, A.A. (2016) Journal of Modern Physics, 7, 1228-1246.

[68] Antonov, A.A. (2016) Dark Matter and Dark Energy are the Invisible Multiverse. American Journal of Modern Physics. http://www.sciencepublishinggroup.com/news/sciencepgfrontiersinfo?articleid=93

[69] Antonov, A.A. (2016) Journal of the Russian Physical and Chemical Society, 87, 63-76. (In Russian) http://www.rusphysics.ru/files/Antonov.Obsor.pdf

[70] Antonov, A.A. (2016) Applied Physics Research, 9, 30-41. https://doi.org/10.5539/apr.v9n2p30

[71] Kantor, I.L. and Solodovnikov, A.S. (1989) Hypercomplex Numbers. Springer-Verlag, Berlin. https://doi.org/10.1007/978-1-4612-3650-4

[72] Antonov, A.A. (2015) Global Journal of Science Frontier Research: A. Physics and Space Science, 15, 6-15.

[73] Antonov, A.A. (2018) Journal of Modern Physics, 9, 14-34. https://doi.org/10.4236/jmp.2018.91002

[74] Antonov, A.A. (2016) Global Journal of Science Frontier Research: A Physics and Space Science, 16, 4-12.

[75] Antonov, A.A. (2019) Journal of Modern Physics, 10, 1006-1028. https://doi.org/10.4236/jmp.2019.108067

[76] Dyson, F.W., Eddington, A.S. and Davidson, C. (1920) Philosophical Transactions of the Royal Society A: Mathematical, Physical and Engineering Sciences, 220, 291-333. 